\title{
PHYSICAL THERAPY AND FUNCTIONAL REHABILITATION IN PATIENTS WITH HAEMOPHILIC ARTHROPATHY SURGI- CALLY TREATED
}

Dan V. Poenaru ${ }^{a}$

Diana Andrei-Popa ${ }^{1, b}$

Jenel Marian Patrascu ${ }^{a}$

Bogdan Andor ${ }^{a}$

Manuel Oprea ${ }^{a}$

Elena Amaricai ${ }^{b}$

\author{
Original scientific paper
}

$2^{\text {nd }}$ Clinic of Orthopaedics and Traumatology, University of Medicine and Pharmacy "Victor Babes" Timisoara $^{a}$

Department of Balneology Medical Rehabilitation and Rheumatology, University of Medicine and Pharmacy

"Victor Babes" Timisoarab

Received: 04.07.2016

Accepted: 09.08.2016

\begin{abstract}
Patients with haemophilia type A or B may develop, over time, haemophilic arthropathy with different degrees of joint dysfunction. This disorder is a consequence of repeated episodes of intraarticular bleeding, with either spontaneous or traumatic aetiology. In the recent years, the therapeutic management of these patients has changed, still, without prompt early diagnosis and prophylactic treatment, the joints deteriorate to such a degree that only a complex multi-disciplinary approach can offer an optimal outcome. Modern high resolution MRI and prophylaxis treatment can detect and delay early signs of haemophilic arthropathy, but, not all patients have access to these types of early interventions. As a result, there are still patients presenting with different of degrees haemophilic arthropathy, which require surgical treatment. Despite the use of modern, minimal invasive approaches, surgical treatment alone can't offer a good symptom relief and can't provide a good functional outcome. Thus, the integration of physical therapy and functional rehabilitation in the therapeutic scheme can provide a good support in order for these patients to be socio-economically re-integrated. Keywords: haemophilic arthropathy; synovectomy; functional rehabilitation; physical therapy;
\end{abstract}

Conflict of Interests: The authors declare that they have no conflict of interests.

\section{INTRODUCTION}

Haemophilia is a recessive $\mathrm{X}$-linked coagulopathy in which normal blood coagulation is affected due to a deficiency of either factor VIII (in type A, found in $85 \%$ of cases) or factor IX (type B, found in $15 \%$ of cases) $[1,2,4]$. Patients with severe haemophilia are particularly symptomatic and may present spontaneous bleedings starting from early childhood. These bleeding can occur in the musculoskeletal system (representing $80-90 \%$ of bleeding episodes) and less frequently within the mucous or cerebral tissues.

\footnotetext{
${ }^{1}$ Correspondence to:

Diana Andrei-Popa, Department of Balneology Medical Rehabilitation and Rheumatology, University of Medicine and Pharmacy

"Victor Babes" Timisoara

Eftimie Murgu Square no 2, 300041, Timisoara, Romania

E-mail: andreidiana81@gmail.com
} 
Intraarticular bleedings cause synovial hypertrophies and affect the cartilage, progressively deteriorating it and leading to haemophilic arthropathy $[1,2,6]$. The final stage of haemophilic arthropathy is characterized mainly by the presence of marked intraarticular fibrosis, muscular atrophy, inflammatory arthritis, bone mineral density decreasing and formation of subchondral cysts $[4,5]$.

Haemophilic arthropathy can lead to pain, articular deformity and disability. The structural deterioration of the joints can result from a low number of haemarthrosis episodes in patients with a predisposition for arthrosis or it can arise from multiple bleeding episodes $[4,6,7]$.

The objective of this study is to evaluate the effects of physical therapy and functional rehabilitation in patients diagnosed with haemophilic arthropathy who were surgically treated.

The results presented are from cases treated in the Haemophiliac Osteoarticular Surgery Department from 2nd Ortho- paedics and Traumatology Clinic Timisoara in a 15-year period from its inauguration. This department was established in 2001, financed through a special fund from Romania's Health Ministry for the treatment of haemophiliacs.

\section{MATERIAL AND METHODS}

Between 2001 and 2016 there were admitted and treated a number of 177 patients coming from the whole country. A number of 122 patients underwent a total of 123 surgical treatments. The patients' age ranged between 9 and 61 years old.

The treatment protocol, especially the factor substitution, was done in collaboration with a team of specialist physicians from the Pediatric Onco-Haemathology Clinic "Louis Turcanu" Pediatric Hospital Timisoara.

The cases included in this paper are presented in tables 1 and figures 1 and 2 .

Table 1. Cases admitted and treated in the 2nd Orthopaedics and Traumatology Clinic

\begin{tabular}{lc}
\hline Admitted and treated & 177 \\
Patients that underwent surgical treatment & 122 \\
Number of surgical treatments & 123 \\
Haemophilia A & 110 \\
Haemophilia B & 2 \\
Acquired Von Willebrand disease & 1 \\
Infection with VHC and/or VHB & 47 \\
Osteoporosis, osteopenia & 52 \\
\hline
\end{tabular}

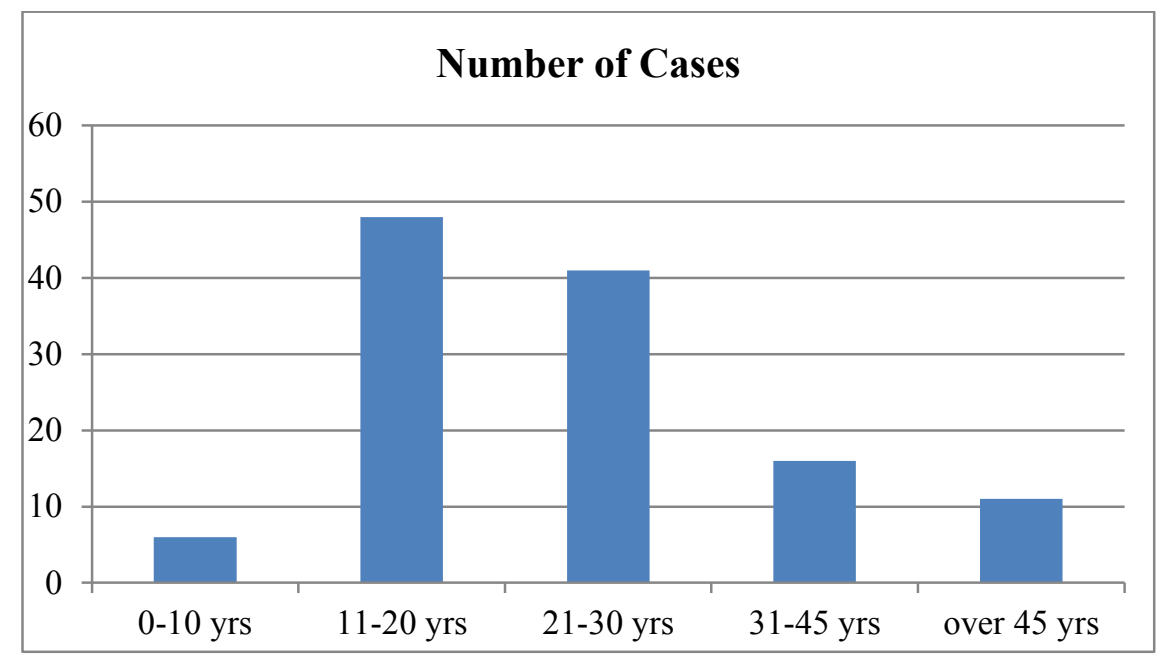

Figure 1. Age-group distribution 


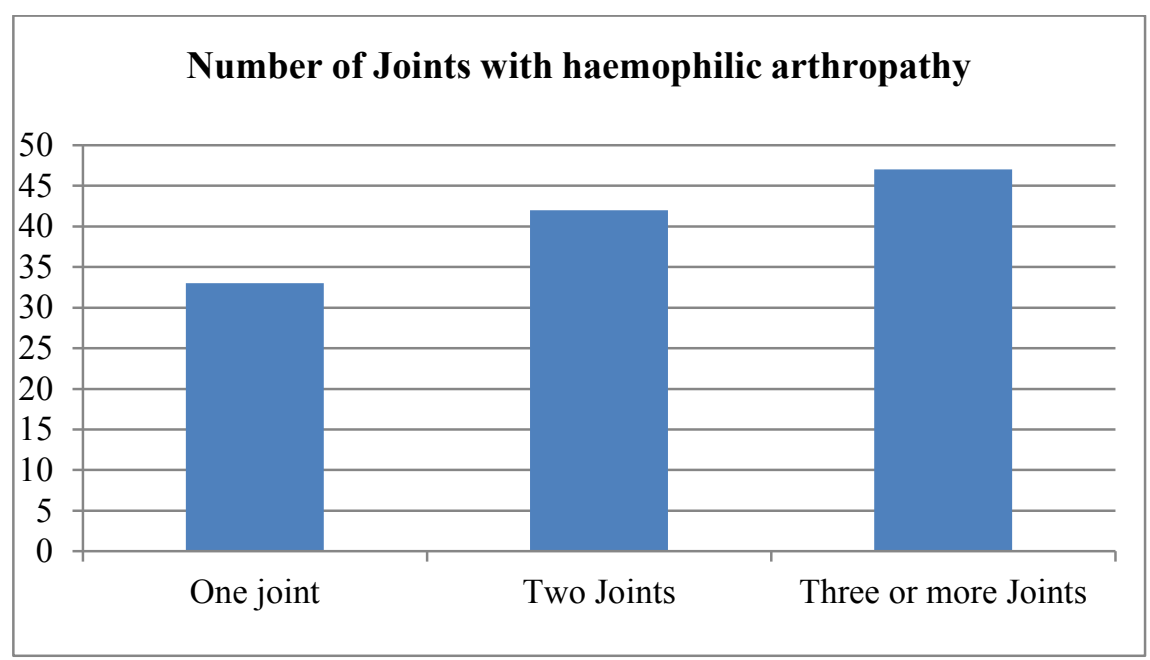

Figure 2. Case distribution in relation with the number of affected joints.

After surgery all patients followed a two-stages rehabilitation program: immediately after surgery (within the 2nd Clinic of Orthopaedics and Traumatology) and another one on medium to long term (within "Cristian Serban" Clinical Centre for Evaluation and Rehabilitation).

In the early postoperatory phase the targeted objectives were improvement of ROM (Range Of Motion) with progressive weight-bearing, gait re-education and regaining of previous abilities. For each patient, a customized physical therapy program was established, consisting of active-passive and active exercises.

Once the patient was discharged from the Orthopaedic Clinic, he was further redirected towards a specialized rehabilitation centre accustomed with treating haemophiliacs. The rehabilitation therapy consists in: electrotherapy, manual massage, exercise program and aquatic therapy.

Electrotherapy consisted in application of low frequency electric currents (with impulses: TENS in cases with articular pain; rectangular currents for strengthening of hypotrophied muscles), medium frequency currents (interferential currents for improving joints mobility, articular pain reduction or muscular decontracture) and high frequency currents (ultrasound in continuous or pulsed form, low intensity; short waves for oedema and haematoma resorption, to reduce inflammation, to stimulate fibrin and col- lagen deposits). Magnetotherapy was utilised for its antalgic effects, to normalize muscular tonus and to improve bone metabolism.

The manual massage types used were relaxing, decontracturant (effleurage, friction and vibration) targeting joints with haemophilic arthropathy. The other type of massage was for stimulation, sthrengthening (petrissage and tapotement) targeting the hypotrophic muscles.

Exercise programme consisted in groups of exercises for maintaining and improving joint ROM, improving muscular strength and endurance, coordination of movements, body posture and alignment, optimization of exercise capacity and, not least, whole body harmonious development. Physical exercises were guided after certain rules: slow, rhythmic execution, without sudden moves; regarding ROM, the progression was slow; whole muscle tonification, respecting the pauses between exercises, proportional with the contraction force.

Aquatic therapy targeted muscular spasm and pain reduction, restoration of muscular strength, attenuation of articular stiffness, improvement of joints ROM and regaining of functional abilities. Water served in the same time both as a recreational and sports activity. Swimming is the most recommended sport for haemophiliacs, maintaining joint's mobility and improving the patient's cardiovascular and pulmonary performances [5]. 
Table 2. Types of surgical interventions performed in patients with haemophilia

Arthroscopic knee synovectomy

Total knee arthroplasty (TKA)

Knee arthrodesis

TKA revision surgery

Septic knee debridement

Elbow synovectomy

Radial head resection

Total Hip Arthroplasty

Arthroscopic ankle synovectomy

Ankle arthrodesis

Cubital Nerve neurolysis

Fracture reduction and fixation

Haemophilic pseudotumour resection

Supracondylar femoral osteotomy

Hip haematoma (infected and fistulised THA: debridement, fistulectomy and lavage)

Distal 1/3 thigh amputation

Other: osteosynthesis material removal

\section{DISCUSSIONS AND CONCLUSIONS}

Even though haemophilic arthropathy is a rare condition, physical therapy and functional rehabilitation plays an important role in the treatment of haemophiliacs with or without subsequent surgery. Rehabilitation is performed with respect of the current principles available for these patients with a high risk for haemorrhage. This therapy can only be performed under strict haemostasis control.

Although worldwide the number of patients with haemophilic arthropathy has remarkably declined as a result of prompt, early substitute treatment, for an optimum duration of time and in sufficient quantities, in Romania the situation is quite different. The treatment with cryoprecipitate and/or plasma tardily initiated, in hospital-stay conditions and under-dosed has led to the expression of severe progressive haemophilic arthropathies that need early surgical treatment. The substitution therapy has been implemented in Romania from 2014 in doses of 1 IU/capita. In these conditions, physical therapy and rehabilitation plays an important role in the comprehensive treatment approach for these patients.

\section{REFERENCES}

1.Agapidou, A., Stavrakis, T., Vlachaki, E., Anagnostis, P, \& Vakalopoulou, S. (2016). The Role of Angiogenesis in Haemophilic Arthropathy: Where Do We Stand and Where Are We Going? Turk J Haematol. 5;33(2):8893. doi: 10.4274/tjh.2016.0031. PubMed PMID: 27211044

2.Dan, V. Poenaru, Margit Şerban, Ioan Branea. (2005). Artropatiile hemofilice. București: Editura Academiei Române

3.Dunn, AL. (2011). Pathophysiology, diagnosis and prevention of arthropathy in patients with haemophilia. Haemophilia 17:571-578.

4.Luck, J.V. Jr, Silva, M., Rodriguez-Merchan, E.C., et al. (2004). Hemophilic arthropathy. J Am Acad Orthop Surg. 12:234-245.

5.M.Serban, H.Ionita, D.Poenaru, L.Ritli. (2009). O viata cu Hemofilie. Timişoara: Ed.Brumar, ISBN: 978-973602-427-6

6.M. Şerban, H. Ioniţă, D.Baghiu, E. Boeriu, A. Cucuianu, D. Mihailov, D. Poenaru, L. Pop, J. Puiu, L. Ritli, V. Uscătescu, D. Lighezan. (2009). Ghid de diagnostic şi tratament în sindroamele hemoragice. Timişoara: Ed.Brumar

7.Rodriguez-Merchan EC, Jimenez-Yuste V, Aznar JA, et al. (2011). Joint protection in haemophilia. Haemophilia. 17 Suppl 2:1-23.

8.Valentino, L.A. (2010). Blood-induced joint disease: the pathophysiology of hemophilic arthropathy. J Thromb Haemost. 8:1895-902. 